\title{
On groups of projective collineations in a space of $K$-spreads.
}

\author{
By Kentaro Yano and Hitosi Hiramatu
}

(Received July 29, 1953)

\section{§ 0. Introduction.}

In an affine space of paths :

$$
\begin{aligned}
& \frac{d^{2} x^{i}}{d s^{2}}+I_{j k}^{i} \frac{d x^{j}}{d s} \frac{d x^{k}}{d s}=0, \\
& (a, b, c, \cdots, i, j, k, \cdots=1,2, \cdots, N)
\end{aligned}
$$

an affine collineation is defined as a point transformation which carries any path into a path and preserves the affine character of the parameter $s$ on it. It was first shown by L. P. Eisenhart and M. S. Knebelman ([5], [6]) that a necessary and sufficient condition that an infinitesimal point transformation

$$
\bar{x}^{i}=x^{i}+\xi^{i}(x) d t
$$

be an affine collineation is that $\xi^{i}$ satisfy

$$
\xi^{i}{ }_{j, k}+\xi^{a} \Gamma_{{ }_{j, a},}^{i}-\xi^{i}, a \Gamma_{j k}^{a}+\xi^{a},{ }_{j} \Gamma_{a k}^{i}+\xi^{a}{ }_{, k} \Gamma_{j a}^{i}=0,
$$

where the comma followed by an index denotes partial differentiation with respect to $x^{i}$.

But, if we denote the Lie derivative ([9], [15]) of a geometric object $\Omega$ with respect to the infinitesimal point transformation $(0.2)$ by $X \Omega$, then condition $(0.3)$ can be rewritten in a very simple form

$$
X \Gamma_{j k}^{i} \equiv \xi_{; j ; k}^{i}+R^{i}{ }_{j k l} \xi^{l}=0 \text {, }
$$

where the semi-colon followed by an index denotes covariant differentiation with respect to $\Gamma_{j k}^{i}$.

It is well known that the maximum order of a group of affine collineations in an $N$-dimensional space of paths is $N^{2}+N$ and if the space admits a group of affine collineations of the maximum order, then the space is necessarily affinely flat ([5], [15]). 
Now, in a space of paths, a projective collineations is defined as a point transformation which carries any path into a path, the affine parameter on it being not necessarily preserved. A necessary and sufficient condition that $(0.2)$ be a projective collineation is that

$$
X \Gamma_{j k}^{i}=\delta_{j}^{i} \varphi_{k}+\delta_{k}^{i} \varphi_{j},
$$

where $\phi_{k}$ is a certain covariant vector, or that

$$
X \Pi_{j k}^{* i}=0,
$$

where $\Pi_{j k}^{* i}$ are T.Y. Thomas' parameters of projective connexion:

$$
\Pi_{j k}^{* i}=\Gamma_{j k}^{i}-\frac{1}{N+1}\left(\delta_{j}^{i} \Gamma_{a k}^{a}+\delta_{k}^{i} \Gamma_{a j}^{a}\right) .
$$

Since the transformation law of $\Pi_{j k}^{* i}$ under a coordinate transformation is more complicated than that of $\Gamma_{j k}^{i}$, the defining equation of $X \Pi_{j k}^{* i}$ is more complicated than that of $X \Gamma_{j k}^{i}$. So, it seems to the present authors that it is more convenient to use (0.5) than to use (0.6) in the discussions of projective collineations.

It is well known that the maximum order of a group of projective collineations in an $N$-dimensional space of paths is $N^{2}+2 N$ and if the space admits a group of projective collineations of the maximum order, then the space is necessarily projectively flat, ([15]).

J. Douglas ([3]) developed the geometry of general space of paths, the paths being defined by a system of differential equations of the form

$$
\frac{d^{2} x^{i}}{d s^{2}}+\Gamma^{i}\left(x, \frac{d x}{d s}\right)=0,
$$

where the functions $I^{i}$ are homogeneous of the second degree in the arguments $\frac{d x^{i}}{d s}$.

Affine and projective collineations in such a general space of paths are defined in exactly the same way as above. The theory of affine and projective collineations in a general space of paths was studied in detail by M. S. Knebelman ([8]).

Subsequently, J. Douglas ([4]) developed also the geometry of a space of $K$-spreads, $K$-spread being a $K$-dimensional subspace defined by a completely integrable system of partial differential equations of the form 


$$
\begin{gathered}
\frac{\partial^{2} x^{i}}{\partial u^{\beta} \partial u^{\gamma}}+H_{\beta \gamma}^{i}(x, p)=0, \quad\left(p_{\alpha}^{i}=\frac{\partial x^{i}}{\partial u^{\alpha}}\right) \\
(\alpha, \beta, \gamma, \cdots, \lambda, \mu, \nu, \cdots=1,2, \cdots, K)
\end{gathered}
$$

where the functions $H_{\beta \gamma}^{i}$ are symmetric in $\beta$ and $\gamma$ and form a so.called homogeneous function system of $p_{\alpha}^{i}$ with respect to lower indices, that is, they satisfy generalized Euler relations

$$
p_{\lambda}^{a}\left(\left.H_{\beta \gamma}^{i}\right|_{a} ^{\alpha}\right)=\delta_{\beta}^{\alpha} H_{\lambda \gamma}^{i}+\delta_{\gamma}^{\alpha} H_{\beta \lambda}^{i},
$$

the symbol $\left.\right|_{a} ^{\infty}$ denoting partial differentiation with respect to $p_{a}^{a}$.

It was shown by I. Douglas ([4]) that the components of the affine connexion are given by

$$
\Gamma_{j k}^{i}=\left.\left.\frac{1}{K(K+1)} H_{\beta \gamma}^{i}\right|_{j} ^{\beta}\right|_{k} ^{\gamma}
$$

Now, an affine collineation in a space of $K$-spreads is defined as a point transformation which carries any $K$-spread into a $K$-spread, the parameters at corresponding points being related by affine transformations.

A necessary and sufficient condition that a point transformation

$$
\bar{x}^{i}=f^{i}(x)
$$

be an affine collineation is that

$$
\bar{H}_{\beta \gamma}^{i}(\bar{x}, \bar{p})=H_{\beta \gamma}^{i}(\bar{x}, \bar{p}),
$$

where $\bar{H}_{\beta \gamma}^{i}(\bar{x}, \bar{p})$ denote the components of $H_{\beta \gamma}^{i}$ in the coordinate system $\left(\bar{x}^{i}\right)$ when we regard (0.11) as a coordinate transformation and

$$
\bar{p}_{\alpha}^{i}=\frac{\partial \bar{x}^{i}}{\partial x^{a}} p_{a}^{a},
$$

from which, a necessary and sufficient condition that an infinitesimal point transformation $(0.2)$ be an affine collineation is that

$$
X \Gamma^{i}{ }_{j k}=0 \text {, }
$$

where

(0.14) $X \Gamma_{j k}^{i}=\xi^{i},{ }_{j, k}+\xi^{a} \Gamma_{j k, a}^{i}+\left.\xi^{m}{ }_{a} p_{\alpha}^{a} \Gamma_{j k}^{i}\right|_{m} ^{a}-\xi^{i},{ }_{a} \Gamma_{j k}^{a}+\xi^{a},{ }_{j} \Gamma_{a k}^{i}+\xi^{a}{ }_{, k} \Gamma_{j a}^{i}$.

The theory of affine collineations in a space of $K$-spreads was studied by E. T. Davies ([2]) and Buchin Su ([12], [13]). 
A projective collineation in a space of $K$-spreads is defined as a point transformation which carries any $K$-spread into a $K$-spread, the parameters at corresponding points being not necessarily related by affine transformations.

A necessary and sufficient condition that a point transformation (0.11) be a projective collineation is that

$$
\bar{H}_{\beta \gamma}^{i}(\bar{x}, \bar{p})=H_{\beta \gamma}^{i}(\bar{x}, \bar{p})+\bar{p}_{\alpha}^{i} G_{\beta \gamma}^{\alpha}(\bar{x}, \bar{p}),
$$

where $G_{\beta \gamma}^{\alpha}$ are a certain function system of $p_{\alpha}^{i}$ with respect to three indices $\alpha, \beta$ and $\gamma([4])$, from which, a necessary and sufficient condition that $(0.2)$ be a projective collineation is that

$$
X \Gamma^{i}{ }_{j k}=\delta_{j}^{i} \varphi_{k}+\delta_{k}^{i} \mathcal{\varphi}_{j}+p_{\alpha}^{i} \varphi_{j k}^{\alpha},
$$

where

$$
\phi_{k}=\left.\frac{1}{K(K+1)} G_{\alpha \gamma}^{\alpha}\right|_{k} ^{\gamma}, \quad \phi_{j k}^{\alpha}=\left.\left.\frac{1}{K(K+1)} G_{\beta \gamma}^{\alpha}\right|_{j} ^{\beta}\right|_{k} ^{\gamma} .
$$

If we introduce, following T. Y. Thomas and J. Douglas, the quantities

$$
\begin{aligned}
& I I_{j k}^{* i}=I_{j K}^{i j}-\frac{1}{N+1}\left(\delta_{j}^{i} \Gamma_{a k}^{a}+\delta_{k}^{i} \Gamma_{a j}^{a}\right) \\
& -\frac{1}{N-K} p_{\lambda}^{i}\left(\left.\Gamma_{j k}^{a}\right|_{a} ^{\lambda}-\left.\frac{1}{N+1} \Gamma_{a j}^{a}\right|_{k} ^{\lambda}-\left.\frac{1}{N+1} \Gamma_{a k}^{a}\right|_{j} ^{\lambda}\right),
\end{aligned}
$$

then condition $(0.16)$ is rewritten in a form

$$
X \Pi_{j k}^{\stackrel{*}{*}=0 .} \text {. }
$$

But, as we remarked already, the transformation law of $\Pi_{j k}^{* i}$ under a coordinate transformation is rather complicated and consequently the defining equation of $X I_{j k}^{* i}$ is more complicated than that of $X I^{i}{ }_{j k}$.

Theory of projective collineations in a space of $K$-spreads was studied by R. S. Clark ([1]) and Buchin Su ([11], [14]).

Now, to study the projective geometry of $K$-spreads, almost all the authors have used the quantities $\Pi_{j k}^{* i}$ defined by $(0.17)$.

These quantities give a geometric object which is invariant under the so-called projective change of $\Gamma_{j k}^{i}$. But they are not components of an affine connexion and their transformation law are rather com- 
plicated. Consequently we cannot apply the theory of covariant differentiation in its original beautiful form.

If we translate such a theory in terms of the method of moving frames of E. Cartan, then we can find that to use $I^{i}{ }_{j k}$ means to use the semi-natural frames of reference and to use $\Pi_{j k i}^{* i}$ means to use the natural frames of reference.

In a recent paper ([16]), we have shown how to construct the projective geometry of $K$-spreads using only the semi-natural frames of E. Cartan. In this theory, all the geometric objects have very simple transformation laws under coordinate transformations.

Thus, we believe that, when we study the projective collineations in a space of $K$-spreads, it is also preferable to use the semi-natural frames of reference. The main purpose of the present paper is to study groups of projective collineations in a space of $K$-spreads in the light of work cited above.

\section{$\S 1$. Projective geometry of $K$-spreads.}

Let us consider an $N$-dimensional space of $K$-spreads referred to a coordinate system $\left(x^{i}\right)$, the $K$-spreads being given by a completely integrable system of partial differential equations

$$
\frac{\partial^{2} x^{i}}{\partial u^{\beta} \partial u^{\gamma}}+H_{\beta \gamma}^{i}(x, p)=0, \quad\left(p_{\alpha}^{i}=\frac{\partial x^{i}}{\partial u^{\alpha}}\right)
$$

where $H_{\beta \gamma}^{i}$ are symmetric in lower indices $\beta$ and $\gamma$ and form a homogeneous function system with respect to these indices. It is well known that the functions difined by

$$
\Gamma_{j k}^{i}=\left.\left.\frac{1}{K(K+1)} H_{\mu \nu}^{i}\right|_{j} ^{\mu}\right|_{k} ^{\nu}
$$

are components of an affine connection and if we use these functions, then the differential equations of the $K$-spreads can be written as

$$
\frac{\partial^{2} x^{i}}{\partial u^{\mu} \partial u^{\nu}}+I_{j k}^{i} p_{\mu}^{j} p_{\nu}^{k}=0 \text {. }
$$

When the functions $H_{\mu \nu}^{i}(x, p)$ are such that (1.1) are completely integrable, then a system of $K$-spreads is uniquely determined. But, when a system of $K$-spreads is given, a system of functions $H_{\mu \nu}^{i}$ is not 
uniquely determined. J. Douglas ([4]) has shown that if $\bar{H}_{\mu \nu}^{i}$ and $H_{\mu \nu}^{i}$ give the same system of $K$-spreads, then they should be related by the equations of the form

$$
\bar{H}_{\mu \nu}^{i}=H_{\mu \nu}^{i}+p_{\lambda}^{i} C_{\mu \nu}^{\lambda},
$$

and consequently ${\overline{I^{i}}}_{{ }_{j}}$ and $\Gamma^{i}{ }_{j k}$ by

$$
\bar{\Gamma}_{j k}^{i}=I^{i}{ }_{j k}+\delta_{j}^{i} \lambda_{k}+\delta_{k}^{i} \lambda_{j}+p_{a}^{i} \lambda_{j k}^{\infty},
$$

where

$$
\lambda_{j}=\left.\frac{1}{K(K+1)} C_{\alpha \gamma}^{\alpha}\right|_{j} ^{\gamma}, \quad \lambda_{j k}^{\alpha}=\left.\left.\frac{1}{K(K+1)} C_{\beta \gamma}^{\alpha}\right|_{j} ^{\beta}\right|_{k} ^{\gamma} .
$$

These are so-called projective changes of $H_{\mu \nu}^{i}$ and $I^{i}{ }_{j k}^{i}$ respectively.

We have shown ([16]) that the projective geometry of $K$-spreads is equivalent to the theory of a space of elements $\left(x^{i}, p_{a}^{i}\right)$ with normal projective connexion whose family of $K$-dimensional totally geodesic subspaces is given by (1.1). The components $\Pi_{j_{k}}^{0}, \Pi_{j k}^{0 \alpha}$ and $\Pi_{{ }_{j k}}^{i}$ of this normal projective connexion referred to a semi-natural frame of reference are given by

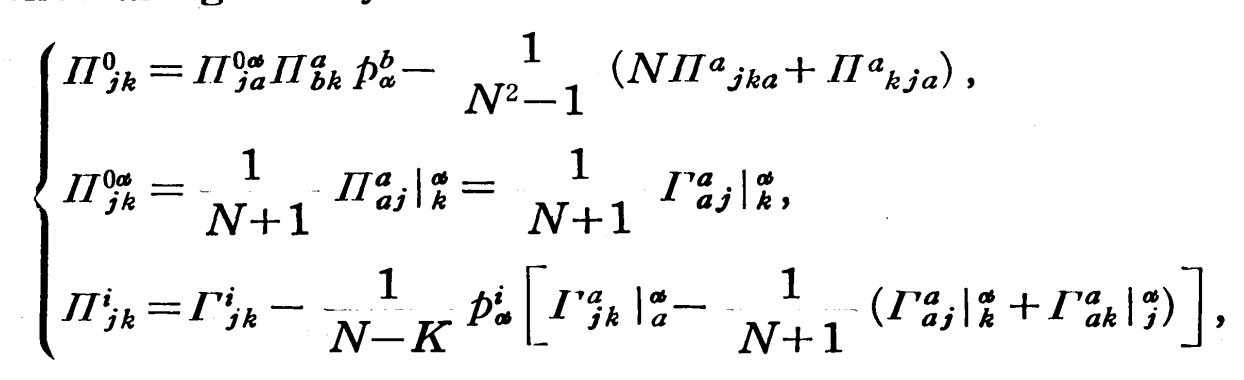

where

$$
\begin{gathered}
\Pi_{j k l}^{i}=\left(\Pi_{j k, l}^{i}-\left.\Pi_{j k}^{i}\right|_{a} ^{a} \Pi_{b l}^{a} p_{a}^{b}\right)-\left(\Pi_{j l, k}^{i}-\left.I I_{j l}^{i}\right|_{a} ^{a} \Pi_{b k}^{a} p_{a}^{b}\right) \\
+\Pi_{j k}^{a} \Pi_{a l}^{i}-\Pi_{j l}^{a} \Pi_{a k}^{i} .
\end{gathered}
$$

It is easily verified that the functions $\Pi_{j k}^{0 \alpha}$ are components of a tensor and the functions $\Pi_{j k}^{i}$ are components of an affine connexion.

Under a projective change (1.4) of $\Gamma_{j k}^{i}$, the functions $\Pi_{j k}^{0}, \Pi_{j k}^{0 \infty}$ and $\Pi_{j k}^{i}$ are transformed into $\bar{\Pi}_{j k}^{0}, \bar{\Pi}_{j k}^{0 x}$ and $\bar{I}_{j k}^{i}$ respectively following the formulae:

$$
\left\{\begin{array}{l}
\bar{\Pi}_{j k}^{0}=\Pi_{j k}^{0}+\lambda_{j, k}-\lambda_{i} \Pi_{j k}^{i}-\lambda_{j} \lambda_{k}, \quad \bar{\Pi}_{j k}^{0 \alpha}=\Pi_{j k}^{0 \alpha}+\left.\lambda_{j}\right|_{k} ^{\alpha}, \\
\bar{\Pi}_{j k}^{i}=\Pi_{i k}^{i}+\delta_{j}^{i} \lambda_{k}+\delta_{k}^{i} \lambda_{j} .
\end{array}\right.
$$


Now, if we define the covariant derivative of a tensor, say, of $T_{j k}^{i}$ by

$$
T_{j k ; l}^{i}=T_{j k, l}^{i}-\left.T_{j k}^{i}\right|_{a} ^{a} \Pi_{b l}^{a} p_{a}^{b}+T_{j k}^{a} \Pi_{a l}^{i}-T_{a k}^{i} \Pi_{j l}^{a}-T_{j a}^{i} \Pi_{k l}^{a},
$$

then the curvature tensors of the projective connexion are given by the following formulae:

$$
\begin{aligned}
& \text { (1.9) } P_{j k l}^{0}=S_{j k ; l}-S_{j l ; k}+\Pi_{j a}^{0 \alpha} \Pi_{b k l}^{a} p_{a}^{b}, \quad\left(S_{j k}=\Pi_{j k}^{0}-\Pi_{j a}^{0 \alpha} \Pi_{b k}^{a} p_{\alpha}^{b}\right) \\
& (1.10) \quad P^{0}{ }_{j k l}^{\alpha}=\left.S_{j k}\right|_{l} ^{\alpha}-\Pi_{j l ; k}^{0 \alpha}+\left.\Pi_{j a}^{0 \beta} \Pi_{b k}^{a}\right|_{l} ^{\alpha} p_{\beta}^{b}, \\
& (1.11) \quad P^{0}{ }_{j k l}^{\alpha \beta}=0, \\
& (1.12) \quad P^{i}{ }_{j k l}=\Pi_{j k l}^{i}+S_{j k} \delta_{l}^{i}-S_{j l} \delta_{k}^{i}-\delta_{j}^{i}\left(S_{k l}-S_{l k}\right), \\
& (1.13) \quad P^{i}{ }_{j k l}^{\alpha}=\left.\Pi_{j k}^{i}\right|_{l} ^{\alpha}-\delta_{j}^{i} \Pi_{k l}^{0 \alpha}-\delta_{k}^{i} I I_{j l}^{0 \alpha},
\end{aligned}
$$

where $P_{j k l}^{j}$ and $P^{i}{ }_{j k l}$ are homogeneous functions of degree zero with respect to $p_{\alpha}^{i}$ and $P^{0}{ }_{j k l}^{\alpha}$ and $P^{i}{ }_{j k l}{ }^{\alpha}$ both form homogeneous function system with respect to the upper index $\alpha$.

We remark here that they satisfy the relations:

$$
\begin{aligned}
& P^{0}{ }_{j k l}^{\alpha} p_{\lambda}^{l}=0, \quad P^{i}{ }_{j k l}{ }^{\alpha} p_{\lambda}^{l}=0, \quad P^{i}{ }_{j k l}^{\alpha}=P^{i}{ }_{k j l}{ }^{\alpha}, \\
& P^{a}{ }_{a k l}{ }^{\alpha}=P^{a}{ }_{j a l}^{\alpha}=P^{\alpha}{ }_{j k a}{ }^{\alpha}=P^{a}{ }_{j k a}=0 .
\end{aligned}
$$

It will be easily seen that the covariant derivative of $p_{a}^{i}$ is identically zero: $p_{\alpha ; k}^{i}=0$.

By a straightforward calculation, we get the following formulae:

$$
\begin{aligned}
& T^{i}{ }_{j k ; l ; m}-T^{i}{ }_{j k ; m ; l}=T^{a}{ }_{j k} \Pi^{i}{ }_{a l m}-T_{a k}^{i} \Pi^{a}{ }_{j l m} \\
& -T^{i}{ }_{j a} \Pi_{k l m}^{a}-\left.T_{j k}^{i}\right|_{a} ^{a} \Pi_{b l m}^{a} p_{a}^{b}, \\
& \left.T^{i}{ }_{j k ; l}\right|_{m} ^{a}-\left.T^{i}{ }_{j k}\right|_{m ; l} ^{a}=\left.T^{a}{ }_{j k} \Pi_{a l}^{i}\right|_{m} ^{a}-\left.T_{a k}^{i} \Pi_{j l}^{a}\right|_{m} ^{\alpha} \\
& -\left.T_{j a}^{i} \Pi_{k l}^{a}\right|_{m} ^{a}-\left.\left.T_{j k}^{i}\right|_{a} ^{\beta} \Pi_{b l}^{a}\right|_{m} ^{a} p_{\beta}^{b}, \\
& \left(\Pi^{i}{ }_{j k l ; m}+\left.\Pi_{j k}^{i}\right|_{a} ^{a} \Pi_{b l m}^{a} p_{a}^{b}\right)+\left(\Pi^{i}{ }_{j l m ; k}+\left.\Pi_{j l}^{i}\right|_{a} ^{a} \Pi_{b m k}^{a_{b a}} p_{a}^{b}\right) \\
& +\left(\Pi_{j m k ; l}^{i}+\left.\Pi_{j m}^{i}\right|_{a} ^{\alpha} \Pi_{b k l}^{a} p_{a}^{b}\right)=0, \\
& \text { (1.18) }\left.\quad \Pi^{i}{ }_{j k l}\right|_{m} ^{a}=\left(\left.\Pi_{j k}^{i}\right|_{m ; l} ^{a}-\left.\left.\Pi_{j k}^{i}\right|_{a} ^{\beta} \Pi_{b l}^{a}\right|_{m} ^{a} p_{\beta}^{b}\right)-\left(\left.\Pi_{j l}^{i}\right|_{m ; k} ^{a}-\left.\left.\Pi_{j l}^{i}\right|_{a} ^{\beta} \Pi_{b k}^{a}\right|_{m} ^{a} p_{\beta}^{b}\right) \text {. }
\end{aligned}
$$

We shall deduce here some formulae which are useful in the following discussions. First of all, from (1.13), (1.18) and 


$$
\left.P^{i}{ }_{j k l}\right|_{m} ^{\alpha}=\left.I I^{i}{ }_{j k l}\right|_{m} ^{a}+\left.S_{j k}\right|_{m} ^{a} \delta_{l}^{i}-\left.S_{j l}\right|_{m} ^{\alpha} \delta_{k}^{i}-\delta_{j}^{i}\left(\left.S_{k l}\right|_{m} ^{\alpha}-\left.S_{l k}\right|_{m} ^{\infty}\right),
$$

we find

$$
\begin{aligned}
\left.P^{i}{ }_{j k l}\right|_{m} ^{\alpha} & =P^{i}{ }_{j k m}{ }^{\alpha}{ }_{; l}+P^{0}{ }_{j k m}{ }^{\alpha} \delta_{l}^{i}-\delta_{j}^{i} P_{k l m}{ }^{\alpha}-P^{i}{ }_{j k a}{ }^{\beta} P^{a}{ }_{b l m}{ }^{\alpha} p_{\beta}^{b}-P^{i}{ }_{j k l}^{\beta} \Pi_{b m}^{0 \alpha} p_{\beta}^{b} \\
& -P^{i}{ }_{j l m}{ }^{\alpha}{ }_{;}-P^{0}{ }_{j l m} \delta_{k}^{i}+\delta_{j}^{i} P^{0}{ }_{l k m}^{\alpha}+P^{i}{ }_{j l a}{ }^{\beta} P^{a}{ }_{b k m}{ }^{\alpha} p_{\beta}^{b}+P^{i}{ }_{j l k}^{\beta} \Pi_{b m}^{0 \alpha} p_{\beta}^{b} .
\end{aligned}
$$

In this equation, we put $i=l$, contract and take account of (1.14) and of $P^{i}{ }_{j k l}^{\alpha}=P_{k j l}^{i}$, then we get

$$
P^{0}{ }_{j k l}^{\alpha}=-\frac{1}{N-1}\left[P^{a}{ }_{j k l}{ }^{\alpha} ; a-P^{c}{ }_{j k a^{\beta}} P^{a}{ }_{b c l}{ }^{\alpha} p_{\beta}^{b}\right],
$$

from which we can see that $P_{j k l}^{0}$ is symmetric in $j$ and $k$ and consequently we have

$$
\left.P_{j k l}^{i}\right|_{m} ^{\alpha}=P_{j k m}^{i}{ }_{j l}^{\alpha}+P_{j k m}^{0}{ }^{\alpha} \delta_{l}^{i}-P^{i}{ }_{j k a}^{\beta} P^{a}{ }_{b l m}{ }^{\alpha} p_{\beta}^{b}-P^{i}{ }_{j k l}^{\beta} \Pi_{b m}^{0 \alpha} p_{\beta}^{b}-(k, l),
$$

where $-(k, l)$ means that we subtract the expressions which are obtained by interchanging the indices $k$ and $l$ in the foregoing terms.

In (1.20), putting $i=m$ and contracting, we find

$$
\left.P^{a}{ }_{j k l}\right|_{a} ^{\alpha}=P^{0}{ }_{j k l}^{\alpha}-P^{c}{ }_{j k a}^{\beta} P^{a}{ }_{b l c}^{\alpha} p_{\beta}^{b}-P^{c}{ }_{j k l}^{\beta} \Pi_{b c}^{0 \alpha} p_{\beta}^{b}-(k, l) \text {. }
$$

Next, we substitute (1.12) and (1.13) into (1.17) and take account of $P^{i}{ }_{j k a}{ }^{\beta} p_{a}^{a}=0$, then we obtain, by the use of (1.9),

$$
\begin{aligned}
P^{i}{ }_{j k l ; m}+P^{i}{ }_{j k a}{ }^{\beta} P^{a}{ }_{b l m}{ }^{\beta} p_{\beta}^{b}- & P^{i}{ }_{j k m}{ }^{\beta} S_{b l} p_{\beta}^{b}+P^{i}{ }_{j k l}{ }^{\beta} S_{b k m} p_{\beta}^{b} \\
& +P^{0}{ }_{j k l} \delta_{m}^{i}+\delta_{j}^{i} P^{0}{ }_{k l m}+c y c(k, l, m)=0,
\end{aligned}
$$

where $+(k, l, m)$ means that we add the terms obtained by a cyclic change of the indices $k, l$ and $m$ in the foregoing terms.

In this equations, putting $i=j$, contracting and taking account of $P a_{a k l}=0$ and of $P a_{a k l}{ }^{\beta}=0$, we find

$$
P_{k l m}^{0}+P^{0}{ }_{l m k}+P_{m k l}^{0}=0,
$$

and consequently

$$
\begin{aligned}
P^{i}{ }_{j k l ; m}+P^{i}{ }_{j k a}^{\alpha} P^{a}{ }_{b l m} p_{a}^{b}-P^{i}{ }_{j k m}{ }^{\beta} S_{b l} p_{\beta}^{b}+P^{i}{ }_{j k l}{ }^{\beta} S_{b m} f_{\beta}^{b} \\
\\
+P^{0}{ }_{j k l} \delta_{m}^{i}+c y c(k, l, m)=0,
\end{aligned}
$$

from which, by the contraction with respect to $i$ and $m$,

$$
\begin{aligned}
P^{a}{ }_{j k l ; a}+(N-2) P^{j}{ }_{j k l} & +P^{c}{ }_{j k a}^{\alpha} P^{a}{ }_{b l c} p_{\alpha}^{b}-P^{c}{ }_{j l a}^{\alpha} P^{a}{ }_{b k c} p_{\alpha}^{b} \\
& +P^{c}{ }_{j k l}{ }^{\alpha} S_{b c} p_{\alpha}^{b}-P^{c}{ }_{j l k}^{\alpha} S_{b c} p_{\beta}^{b}=0 .
\end{aligned}
$$


On groups of projective collineations in a space of $K$-spreads.

\section{§. Lie derivatives.}

In our space of $K$-spreads, we consider an infinitesimal point transformation

$$
\bar{x}^{i}=x^{i}+\xi^{i}(x) d t,
$$

where $\xi^{i}(x)$ is a contravariant vector whose components are functions of a point and $d t$ is an infinitesimal.

We define the Lie differential $D \Omega$ of a geometric object $\Omega$ by

$$
D \Omega=\Omega(\bar{x}, \bar{p})-\bar{\Omega}(\bar{x}, \bar{p})
$$

and Lie derivative $X \boldsymbol{\Omega}$ by

$$
X \Omega=\lim _{d t \rightarrow 0} \frac{D \Omega}{d t},
$$

where $\bar{\Omega}(\bar{x}, \bar{p})$ are components of $\Omega$ in the coordinate system $(\bar{x})$ when we regard (2.1) as a coordinate transformation.

The Lie derivative being thus defined, the Lie derivatives of a scalar $f$, tensor $T_{j k}^{i}$ and projective connexion $I^{i}{ }_{j k}$ are given respectively by

$$
X f=\xi^{a} f_{; a}+\xi a ;\left.b p_{a}^{b} f\right|_{a} ^{\alpha},
$$

(2.5) $X T^{i}{ }_{j k}=\xi^{a} T^{i}{ }_{j k ; a}+\left.\xi^{a}{ }_{; b} p_{a}^{b} T^{i}{ }_{j k}\right|_{a} ^{\alpha}-\xi_{; a}^{i} T^{a}{ }_{j k}+\xi_{; j}^{a} T^{i}{ }_{a k}+\xi_{; k} T^{i}{ }_{j a}$,

(2.6) $X \Pi^{i}{ }_{j k}=\xi^{i} ; j ; k+\xi^{a} ;\left.b p_{a}^{b} l I_{j k}^{i}\right|_{a} ^{\alpha}+I I^{i}{ }_{j k l} \xi^{l}$.

It should be remarked that these Lie derivatives are all tensors and the Lie derivative of $p_{\alpha}^{i}$ is zero: $X p_{\alpha}^{i}=0$.

By a straightforward calculation, we get the following formulae:

(2.7) $X\left(T^{i}{ }_{j k ;}\right)-\left(X T^{i}{ }_{j k}\right)_{; l}=T^{a}{ }_{j k}\left(X \Pi_{a l}^{i}\right)-T^{i}{ }_{a k}\left(X \Pi_{j l}^{a}\right)$

$$
-T^{i}{ }_{j a}\left(X \Pi_{k l}^{a}\right)-\left.T^{i}{ }_{j k}\right|_{a} ^{a}\left(X \Pi_{b l}^{a}\right) p_{a}^{b},
$$

(2.8) $X\left(\left.T^{i}{ }_{j k}\right|_{l}\right)-\left.\left(X T^{i}{ }_{j k}\right)\right|_{l}=0$,

(2.9) $\quad\left(X \Pi_{j k}^{i}\right)_{; l}-\left(X \Pi_{j l}^{i}\right)_{; k}=X \Pi^{i}{ }_{j k l}+\left.I I_{j k}^{i}\right|_{a} ^{\alpha}\left(X \Pi_{b l}^{a}\right) p_{a}^{b}-\left.I I_{j l}^{i}\right|_{a} ^{\alpha}\left(X \Pi_{b k}^{a}\right) p_{a}^{b}$,

(2.10) $\left.\left(X \Pi_{j k}^{i}\right)\right|_{l} ^{\lambda}-X\left(\left.\Pi_{j_{k}}\right|_{l} ^{\lambda}\right)=0$.

Now, when we consider $r$ infinitesimal point transformations

$$
\bar{x}^{i}=x^{i}+\xi_{A}^{i}(x) d t \quad(A, B, C, \cdots=1,2, \cdots, r),
$$


then by a straightforward calculation, we obtain

(2.12) $\left(X_{B} X_{C}\right) f \equiv\left(X_{B} X_{C}-X_{C} X_{B}\right) f=\left(X_{B} \xi_{C}^{a}\right) f_{; a}+\left.\left(X_{B} \xi_{C}^{a}\right)_{; b} p_{a}^{b} f\right|_{a} ^{a}$,

(2.13) $\quad\left(X_{B} X_{C}\right) T^{i}{ }_{j k}=\left(X_{B} \xi_{C}^{a}\right) T^{i}{ }_{j k ; a}+\left.\left(X_{B} \xi_{C}^{a}\right)_{; b} p_{a}^{b} T^{i}{ }_{j k}\right|_{a} ^{a}-\left(X_{B} \xi_{C}^{i}\right)_{; a} T^{a}{ }_{j k}$

$$
+\left(X_{B} \xi_{C}^{a}\right)_{; j} T_{a k}^{i}+\left(X_{B} \xi_{C}^{a}\right)_{; k} T_{j a}^{i},
$$

(2.14). $\left(X_{B} X_{C}\right) \Pi_{j k}^{i}=\left(X_{B} \xi_{C}^{i}\right)_{; j ; k}+\left.\left(X_{B} \xi_{C}^{a}\right)_{; b} p_{\alpha}^{b} \Pi_{j k}^{i}\right|_{a} ^{\infty}+\Pi^{i}{ }_{j k l}\left(X_{B} \xi_{C}^{l}\right)$.

From these equations, we have

THEOREM 2.1. If we apply the operations $\left(X_{B} X_{C}\right)=X_{B} X_{C}-X_{C} X_{B}$ to arbitrary scalar, tensor and affine connexion, then we get the Lie derivatives of these quantities with respect to the vectors $X_{B} \xi_{c}^{i}$.

If $r$ infinitesimal transformations (2.11) are those of an $r$-parameter group of transformations, then we have

$$
\left(X_{B} X_{C}\right) f=C_{B C}^{A} X_{A} f \text {, }
$$

or

$$
X_{B} \xi_{C}^{i}=C_{B C}^{A \xi_{\mathrm{A}}^{i},}
$$

where $C_{B C}{ }^{A}$ are constants of structure of the group.

Thus, substituting $(2.16)$ into (2.12), (2.13) and (2.14), we find

$$
\begin{aligned}
& \left(X_{B} X_{C}\right) f=C_{B C}{ }^{A} X_{A} f, \\
& \left(X_{B} X_{C}\right) T^{i}{ }_{j k}=C_{B C}{ }^{A} X_{A} T^{i}{ }_{j k}, \\
& \left(X_{B} X_{C}\right) \Pi_{j k}^{i}=C_{B C}{ }^{A} X_{A} \Pi_{j k}^{i},
\end{aligned}
$$

and consequently we have

THEOREM 2.2. If $X_{B}$ are $r$ infinitesimal operators of an $r$-parameter group of transformations, then (2.17) are valid not only for a scalar but also for arbitrary tensor and affine connexion.

\section{$\S 3$. Projective collineations.}

In our space of $K$-spreads, we consider a point transformation

$$
\bar{x}^{i}=\bar{x}^{i}\left(x^{1}, x^{2}, \cdots, x^{N}\right) \quad|\partial \bar{x} / \partial x| \neq 0 .
$$

By this transformation, the solutions of

$$
\frac{\partial^{2} x^{i}}{\partial u^{\mu} \partial u^{\nu}}+H_{\mu \nu}^{i}(x, p)=0
$$


are transformed into the solutions of

$$
\frac{\partial^{2} \bar{x}^{i}}{\partial u^{\mu} \partial u^{\nu}}+H_{\mu \nu}^{i}(\bar{x}, \bar{p})=0 \quad\left(\bar{p}_{\alpha}^{i}=\frac{\partial \bar{x}^{i}}{\partial u^{\alpha}}\right) .
$$

On the otherhand, if we regard (3.1) as a coordinate transformation, then the original system of partial differential equations are written also in the form

$$
\frac{\partial^{2} \bar{x}^{i}}{\partial u^{\mu} \partial u^{\nu}}+\bar{H}_{\mu \nu}^{i}(\bar{x}, \bar{p})=0,
$$

where $\bar{H}_{\mu \nu}^{i}(\bar{x}, \bar{p})$ are components of $H_{\mu \nu}^{i}$ in the new coordinate system $\left(\bar{x}^{i}\right)$.

Now, if the system of $K$-spreads defined by (3.2) and that defined by (3.3) coincide, then we call (3.1) a projective collineations in our space of $K$-spreads.

This is the usual definition of projective collineation. But, in this paper, we shall adopt another definition of projective collineation. The system of partial differential equations gives a normal projective connexion with respect to which the solutions of (3.2) are totally geodesic and the system (3.3) gives another projective connexion with respect to which the solutions of (3.3) are totally geodesic. If these projective connexions are projectively equivalent, that is, if we have equations of the form

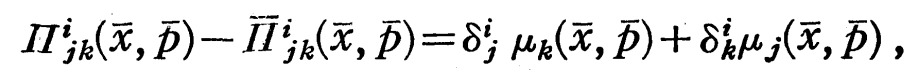

then, we call (3.1) a projective collineations in our space of $K$-spreads, where $\mu_{j}(\bar{x}, \bar{p})$ are some functions of $\bar{x}^{i}$ and $\bar{p}_{a}^{i}$ homogeneous of degree zero with respect to $\bar{p}_{\alpha}^{i}$.

Now, we consider an infinitesimal point transformation

$$
\bar{x}^{i}=x^{i}+\xi^{i}(x) d t .
$$

A necessary and sufficient condition that (3.5) be a projective collineation is that we have

$$
X \Pi_{j k}^{i}=\delta_{j}^{i} \lambda_{k}+\delta_{k}^{i} \lambda_{j},
$$

where $\lambda_{j}$ are homogeneous functions of degree zero with respect to $p_{\alpha}^{i}$ and are components of a covariant vector.

If we eliminate $\lambda_{j}$ from (3.6), we obtain 


$$
X I_{j k}^{* i}=0, \quad \Pi_{j k}^{* i}=\Pi_{j k}^{i}-\frac{1}{N+1}\left(\delta_{j}^{i} \Pi_{a k}^{a}+\delta_{k}^{i} \Pi_{a j}^{a}\right) .
$$

Conversely, if we have (3.7), then $X \Pi_{j k}^{i}$ should have the form (3.6), Thus we have

THEOREM 3.1 In order that an infinitesimal point transformation (3.5) be a projective collineation, it is necessary and sufficient that we have (3.6) or (3.7).

Now, we suppose that the point transformation (3.5) is a projective collineation and that we have chosen a coordinate system in which the vector $\xi^{i}$ has the components $\delta_{1}^{i}$, then (3.7) becomes $I_{j \ldots, 1}^{* i}=0$, and consequently $I_{j k}^{* i}$ do not depend on $x^{1}$.

Conversely, if we suppose that, in a coordinate system, the functions $\Pi_{j k i}^{* i}$ do not depend on $x^{1}$, then for a point transformation $\bar{x}^{i}=x^{i}+\delta_{l}^{i} t$ we have $\Pi_{j_{k}^{*}}^{*}(\bar{x}, \bar{p})-\bar{I}_{j_{k}}^{*}(\bar{x}, \bar{p})=0$, and consequently the space admits a one-parameter group of projective collineations generated by the infinitesimal transformation $\bar{x}^{i}=x^{i}+\delta_{1}^{i} d t$.

We suppose again that the point transformation (3.5) is a projective collineation and that we have chosen a coordinate system in which the vector $\xi^{i}$. has the components $x^{i}$, then (3.7) becomes $\Pi_{j k, a}^{* i} x^{a}+\Pi_{j k}^{* i}=0$ and consequently $\Pi_{j k}^{* i}$ are homogeneous functions of the degree -1 of $x^{i}$.

Conversely, if we suppose that, in a coordinate system, the functions $\Pi_{j k}^{* i}$ are homogeneous of degree -1 of $x^{i}$, then, for a point transformation $\bar{x}^{i}=x^{i} t$, we have $\Pi_{j k}^{* i}(\bar{x}, \bar{p})-\bar{I}_{j k}^{* i}(\bar{x}, \bar{p})=0$ and consequently:the space admits a one-parameter group of projective collineations generated by the infinitesimal transformation $\bar{x}^{i}=x^{i}(1+d t)$. Thus we obtain

THEOREM 3.2. If the space admits an infinitesimal projective collineations, then the space admits also a one-parameter group of projective, collineations generated by the infinitesimal projective collineation.

THEOREM 3.3. In order that the space admit a one-parameter group of projective collineations, it is necessary and sufficient that there exist a coordinate system in which $\Pi_{j k, 1}^{* i}=0$ or $\Pi_{j k, a}^{* i} x^{a}+\Pi_{j k}^{* i}=0$.

Now, we suppose that the space admits $r$ infinitesimal projective collineations

$$
\bar{x}^{i}=x^{i}+\xi_{A}^{i}(x) d t,
$$


then, we have

$$
X_{A} \Pi_{j k}^{i}=\delta_{j}^{i} \lambda_{A k}+\delta_{k}^{i} \lambda_{A j},
$$

from which, we have

THEOREM 3.4. If $\xi_{A}^{i}$ define $r$ infinitesimal projective collineations, then $c^{A} \xi_{A}^{i}$ defines also an infinitesimal projective collineation, where c's are constants.

Now, in general, we have, from Theorem 2.1,

$$
\left(X_{B} X_{C}\right) \Pi_{j k}^{i}=\left(X_{B} \xi_{C}^{i}\right)_{; j ; k}+\left.\left(X_{B} \xi_{C}^{a}\right)_{; b} p_{a}^{b} \Pi_{j k}^{i}\right|_{a} ^{\alpha}+\Pi_{j k l}^{i}\left(X_{B} \xi_{C}^{l}\right) \text {. }
$$

On the other hand, if $X_{A} f$ define projective collineations, then we have

$$
X_{C} \Pi_{j k}^{i}=\delta_{j}^{i} \lambda_{C k}+\delta_{k}^{i} \lambda_{C j},
$$

from which,

$$
\left(X_{B} X_{C}\right) \Pi_{j k}^{i}=\delta_{j}^{i}\left(X_{B} \lambda_{C k}-X_{C} \lambda_{B k}\right)+\delta_{k}^{i}\left(X_{B} \lambda_{C j}-X_{C} \lambda_{B j}\right)
$$

Thus we have

THEOREM 3.5. If $\xi_{A}^{i}$ define $r$ infinitesimal projective collineations, then $X_{B} \xi_{C}^{i}$ define also infinitesimal projective collineations.

If $X_{A} f$ are $r$ generators of an $r$-parameter group of transformations, then this group consists of transformations which belongs to a oneparameter group of transformations generated by $c^{A} X_{A} f$ and of those which are product of these transformations. Thus we have

THEOREM 3.6. If each of $r$ generators of an $r$-parameter group of transformations is the generator of one-parameter group of projective collineations, then every transformation of this group is a projective collineation.

Moreover, by the second fundamental theorem of Lie and Theorem 3.6, we obtain

THEOREM 3.7. If $X_{A} f$ are complete set of generators of oneparameter group of projective collineations, then these are generators of an r-parameter group of projective collineations.

\section{$\S 4$. Integrability conditions.}

In this section, we shall examine the conditions that equations

$$
X \Pi_{j k}^{i}=\xi_{; j ; k}^{i}+\left.\xi^{a}{ }_{; b} p_{a}^{b} \Pi_{j k}^{i}\right|_{a} ^{\alpha}+\Pi_{j k l}^{i} \xi^{l}=\delta_{j}^{i} \lambda_{k}+\delta_{k}^{i} \lambda_{j}
$$


admit solutions $\xi^{i}$ and $\lambda_{j}$, $\xi^{i}$ being functions of $x^{i}$ only and $\lambda_{j}$ being homogeneous functions of degree zero of $p_{\alpha}^{i}$.

From (2.9) and (4.1), we find

$$
\lambda_{j ; k}=X S_{j k}+\Pi_{j k}^{0 \lambda} \lambda_{a} p_{\lambda}^{a}
$$

by virtus of

$$
P{ }_{j k a}^{\lambda}=\left.\Pi_{j k}^{a}\right|_{a} ^{\lambda}-I I_{j k}^{0 \lambda}-\Pi_{k j}^{0 \lambda}=0 .
$$

From (2.10) and (4.1), we find

$$
\left.\lambda_{j}\right|_{k} ^{\lambda}=\frac{1}{N+1} X\left(\left.\Pi_{a j}^{a}\right|_{k} ^{\lambda}\right)=X \Pi_{j k}^{0 \lambda} .
$$

Thus, we may consider a system of linear partial differential equations

$$
\left\{\begin{array}{l}
\xi_{; j}^{i}=\xi_{j}^{i}, \\
\left.\xi^{i}\right|_{k} ^{\alpha}=0, \\
\xi_{j ; k}^{\alpha}=-\left.\xi \xi_{b} p_{\alpha}^{b} \Pi_{j k}^{i}\right|_{a} ^{\alpha}-I I^{i}{ }_{j k l} \xi l+\delta_{j}^{i} \lambda_{k}+\delta_{k}^{i} \lambda_{j}, \\
\left.\xi^{i}\right|_{k} ^{\alpha}=\left.\xi^{a} \Pi_{a j}^{i}\right|_{k} ^{\alpha}, \\
\lambda_{j ; k}=X S_{j k}+\Pi_{j k}^{0 \alpha} \lambda_{a} p_{a}^{a}, \\
\left.\lambda_{j}\right|_{k} ^{\alpha}=X \Pi_{j k}^{0 \alpha} .
\end{array}\right.
$$

If these equations admit solutions $\xi^{i}$ and $\lambda_{j}$, then $\xi^{i}$ do not contain $p_{a}^{i}$ and $\lambda_{j}$ are homogeneous functions of degree zero of $p_{a}^{i}$, because we have

$$
\left(\lambda_{j} \mid \begin{array}{l}
\alpha \\
k
\end{array}\right) p_{\beta}^{k}=\left(X \Pi_{j k}^{0 \alpha}\right) p_{\beta}^{k}=X\left(I_{j k}^{0 \alpha} p_{\beta}^{k}\right)=0 .
$$

Now, from (2.9) and (4.1), we find

$$
X P^{i}{ }_{j k l}=-P^{i}{ }_{j k l}^{\alpha} \lambda_{a} p_{a}^{a}+P^{i}{ }_{j l k}^{\alpha}{ }^{\alpha} \lambda_{a} p_{a}^{a} .
$$

From (2.10) and (4.1), we obtain

$$
X P^{i}{ }_{j k l}{ }^{\lambda}=0 \text {. }
$$

Next, substituting (4.2) and (4.3) into

$$
\left.\lambda_{j ; k}\right|_{l} ^{a}-\left.\lambda_{j}\right|_{l ; k} ^{a}=-\left.\lambda_{a} \Pi_{j k}^{a}\right|_{l} ^{a}-\left.\left.\Pi_{b k}^{a}\right|_{l} ^{a} p_{\beta}^{b} \lambda_{j}\right|_{a} ^{\beta},
$$

taking account of

$$
\begin{gathered}
\left(X I I_{j l}^{0 \alpha}\right)_{; k}=X\left(\Pi_{j l ; k}^{0 \alpha}\right)+\Pi_{j l}^{0 \alpha} \lambda_{k}+\Pi_{j k}^{0 \alpha} \lambda_{l}+\lambda_{j} \Pi_{k l}^{0 \alpha}+\left.\Pi_{j l}^{0 \alpha}\right|_{k} ^{\beta} \lambda_{a} p_{\beta}^{a}, \\
\left.\Pi_{j l}^{0 \alpha}\right|_{k} ^{\beta}=\left.I I_{j k}^{0 \beta}\right|_{l} ^{\infty}
\end{gathered}
$$


On groups of projective collineations in a space of $K$-spreads.

145

and

we find

$$
X P^{i}{ }_{j k l}^{\alpha}=\left.X \Pi_{j k}^{i}\right|_{l} ^{\alpha}-\delta_{j}^{i} X \Pi_{k l}^{0 \alpha}-\delta_{k}^{i} X \Pi_{j l}^{0 \alpha}=0,
$$

$$
X P^{0}{ }_{j k l}^{\infty}=-\lambda_{a} P^{a}{ }_{j k l}{ }^{\infty} .
$$

Applying the formula (2.7) to the tensor $S_{j k}$, we find

$$
X\left(S_{j k ; l}\right)-\left(X S_{j k}\right)_{;}=-S_{a k} X \Pi_{j l}^{a}-S_{j a} X \Pi_{k l}^{a}-\left.S_{j k}\right|_{a} ^{a} X \Pi_{b l}^{a} p_{a}^{b},
$$

from which, combined with (4.1) and (4.2),

$$
\begin{aligned}
\lambda_{j ; k ; l}-\Pi_{j k ;}^{0 \alpha} \lambda_{a} p_{a}^{a} & -\Pi_{j k}^{0 \alpha} X S_{a l} p_{a}^{a}-\Pi_{j k}^{0 \alpha} \Pi_{a l}^{0 \beta} \lambda_{b} p_{\beta}^{b} p_{a}^{a}-X\left(S_{j k ; l}\right) \\
& =S_{j k} \lambda_{l}+S_{l k} \lambda_{j}+S_{j a} X \Pi_{k l}^{a}+\left.S_{j k}\right|_{l} ^{\alpha} \lambda_{a} p_{a}^{a} .
\end{aligned}
$$

Subtracting, from this equation, the equation obtained from this by interchanging $k$ and $l$, and taking account of (1.15), we find

$$
\begin{aligned}
X\left(S_{j k ; l}\right. & \left.-S_{j l ; k}\right)+X \Pi_{j a}^{0 \alpha} I_{b k l}^{a} p_{a}^{b} \\
& =-\lambda_{a} P^{a}{ }_{j k l}-\left(\left.S_{j k}\right|_{l} ^{\alpha}-\Pi_{j l ; k}^{\alpha \alpha}\right) \lambda_{a} p_{a}^{a}+\left(\left.S_{j l}\right|_{k} ^{\alpha}-\Pi I_{j k ; l}^{0 \alpha}\right) \lambda_{a} p_{a}^{a} \\
& +\Pi_{j a}^{0 \alpha}\left(X S_{b k} \delta_{l}^{a}-X S_{b l} \delta_{k}^{a}-\delta_{k}^{a} \Pi_{b l}^{0 \beta} \lambda_{c} p_{\beta}^{c}+\delta_{l}^{a} \Pi_{b k}^{0 \beta} \lambda_{c} p_{\beta}^{c}\right) p_{a}^{b}
\end{aligned}
$$

or

$$
\begin{aligned}
X\left(S_{j k ; l}-S_{j l ; k}\right) & +\left(X \Pi_{j a}^{0 \alpha}\right) \Pi_{b k l}^{a} p_{a}^{b} \\
= & -\lambda_{a} P^{a}{ }_{j k l}-\left(\left.S_{j k}\right|_{l} ^{\alpha}-\Pi_{j l ; k}^{0 \alpha}+\left.\Pi_{j a}^{0 \beta} \Pi_{b k}^{a}\right|_{l} ^{\alpha} p_{\beta}^{b}\right) \lambda_{a} p_{a}^{a} \\
& +\left(\left.S_{j l}\right|_{k} ^{\alpha}-\Pi_{j k ; l}^{0 \alpha}+\left.\Pi_{j a}^{0 \beta} \Pi \Pi_{b l}^{a}\right|_{k} ^{\alpha} p_{\beta}^{b}\right) \lambda_{a} p_{a}^{a} \\
& +\Pi_{j a}^{0 \alpha}\left[X S_{b k} \delta_{l}^{a}-X S_{b l} \delta_{k}^{a}-\delta_{b}^{a}\left(X S_{k l}-X S_{l k}\right)\right. \\
& \left.+\left(\left.\Pi_{b k}^{a}\right|_{l} ^{\beta}-\delta_{b}^{a} \Pi_{k l}^{0 \beta}-\delta_{k}^{a} \Pi_{b l}^{0 \beta}\right) \lambda_{c} p_{\beta}^{c}-\left(\left.\Pi_{b l}^{a}\right|_{k} ^{\beta}-\delta_{b}^{a} \Pi_{l k}^{0 \beta}-\delta_{l}^{a} \Pi_{b k}^{0 \beta}\right) \lambda_{c} p_{\beta}^{c}\right] p_{a}^{b} .
\end{aligned}
$$

Adding $\Pi_{j a}^{c a} X \Pi_{b k l}^{a} p_{a}^{b}$ to this equation and taking account of (1.9), (1.10), (1.12), (1.13) and (4.5), we get

$$
X P^{0}{ }_{j k l}=-\lambda_{a} P^{a}{ }_{j k l}-P^{0}{ }_{j k l}{ }^{\alpha} \lambda_{a} p_{a}^{a}+P^{0}{ }_{j l k}{ }^{\alpha} \lambda_{a} p_{a}^{a} .
$$

Thus, the integrability conditions of the system of partial differential equations (4.4) are given by (4.5), (4.6), (4.7), (4.8) and equations obtained from these by successive covariant differentiations with respect to $x^{i}$ and successive partial differentiation with respect to $p_{\alpha}^{i}$.

First of all, we shall show that conditions (4.7) and (4.8) are obtained from (4.5), (4.6) and their successive derivatives. 
Applying the operstor $X$ to both members of (1.19) and taking account of

$$
\begin{aligned}
& X\left(P^{a}{ }_{j k l}{ }^{\alpha} ; a\right)=\left(X P^{a}{ }_{j k l}{ }^{\alpha}\right)_{;}{ }_{a}+P^{c}{ }_{j k l}{ }^{\alpha} X \Pi_{c a}^{a}-P^{a}{ }_{c k l}{ }^{\alpha} X \Pi_{j a}^{c}-P^{a}{ }_{j c l}{ }^{\alpha} X \Pi_{k a}^{c} \\
& -P^{a}{ }_{j k c}{ }^{\alpha} X \Pi_{l a}^{c}-\left.P^{a}{ }_{j k l}{ }^{\alpha}\right|_{c} ^{\beta} X \Pi_{b a}^{c} p_{\beta}^{b} \\
& =\left(X P^{a}{ }_{j k l}^{\alpha}\right)_{; a}+(N-2) \lambda_{a} P^{a}{ }_{j k l}{ }^{\alpha}-\left.\lambda_{a} P^{a}{ }_{j k l}{ }^{\alpha}\right|_{b} ^{\beta} p_{\beta}^{b}-\left.P^{a}{ }_{j k l}{ }^{\alpha}\right|_{a} ^{\beta} \lambda_{b} p_{\beta}^{b} \\
& =\left(X P^{a}{ }_{j k l}{ }^{\alpha}\right)_{; a}+(N-1) \lambda_{a} P^{a}{ }_{j k l}{ }^{a} \text {, }
\end{aligned}
$$

we find

$$
\begin{aligned}
& X P^{0}{ }_{j k l}{ }^{\alpha}+\lambda_{a} P^{a}{ }_{j k l}{ }^{\alpha} \\
& =-\frac{1}{N-1}\left[\left(X P^{a}{ }_{j k l}{ }^{\alpha}\right)_{; a}-X P^{c}{ }_{j k a}{ }^{\beta} P^{a}{ }_{b c l}{ }^{\alpha} p_{\beta}^{b}-P^{c}{ }_{j k a}{ }^{\beta} X P^{a}{ }_{b c l}{ }^{\alpha} p_{\beta}^{b}\right]
\end{aligned}
$$

which shows that condition (4.7) are obtained from (4.6) and its covariant derivatives.

Next, we apply formula (2.7) to the tensor $P^{a}{ }_{j k l}$, then we obtain, using (1.21),

$$
\begin{aligned}
X\left(P^{a}{ }_{j k l ; a}\right)=( & \left.X P^{a}{ }_{j k l}\right)_{; a}+(N-2) \lambda_{a} P^{a}{ }_{j k l}-\left.P^{a}{ }_{j k l}\right|_{a} ^{\beta} \lambda_{b} p_{\beta}^{b} \\
=\left(X P^{a}{ }_{j k l}\right)_{;} a & +(N-2) \lambda_{a} P^{a}{ }_{j k l}-\left(P^{0}{ }_{j k l}{ }^{\beta}-P^{c}{ }_{j k a}{ }^{\alpha} P^{a}{ }_{b l c}{ }^{\beta} p_{\alpha}^{b}-P^{c}{ }_{j k l}{ }^{\alpha} \Pi_{b c}^{0 \beta} p_{a}^{b}\right) \lambda_{d} p_{\beta}^{d} \\
& +\left(P^{0}{ }_{j l k}{ }^{\beta}-P^{c}{ }_{j l a}{ }^{\alpha} P^{a}{ }_{b k c}{ }^{\beta} p_{\alpha}^{b}-P^{c}{ }_{j l k}{ }^{\alpha} \Pi_{b c}^{0 \beta} p_{\alpha}^{b}\right) \lambda_{d} p_{\beta}^{d} .
\end{aligned}
$$

Applying the operator $X$ to both members of (1.24) and using the above relation, we find

$$
\begin{gathered}
\left(X P^{a}{ }_{j k l}\right)_{; a}+(N-2) \lambda_{a} P^{a}{ }_{j k l}-\left(P^{0}{ }_{j k l}^{\beta}-P^{c}{ }_{j k a}{ }^{\alpha} P^{a} a_{b l c}^{\beta} p_{a}^{b}-P^{c}{ }_{j k l}^{\alpha} \Pi_{b c}^{0 \beta} p_{a}^{b}\right) \lambda_{d} p_{\beta}^{d} \\
+\left(P^{0}{ }_{j l k}^{\beta}-P^{c}{ }_{j l a}^{\alpha} P^{a}{ }_{b k c}^{\beta} p_{\alpha}^{b}-P^{c}{ }_{j l k}^{\alpha} I I_{b c}^{0 \beta} p_{a}^{b}\right) \lambda_{d} p_{\beta}^{d} \\
+(N-2) X P^{0}{ }_{j k l}+X P^{c}{ }_{j k a}^{\beta} P^{a}{ }_{b l c} p_{\beta}^{b}+P^{c}{ }_{j k a}^{\beta} X P^{a}{ }_{b l c} p_{\beta}^{b} \\
+X P^{c}{ }_{j k l}^{\beta} S_{b c} p_{\beta}^{b}+P^{c}{ }_{j k l}^{\beta} X S_{b c} p_{\beta}^{b}-X P^{c}{ }_{j l a}^{\beta} P^{a}{ }_{b k c} p_{\beta}^{b} \\
-P^{c}{ }_{j l a}^{\beta} X P^{a}{ }_{b k c} p_{\beta}^{b}-X P^{c}{ }_{j l k}{ }^{\beta} S_{b c} p_{\beta}^{b}-P^{c}{ }_{j l k}{ }^{\beta} X S_{b c} p_{\beta}^{b}=0 .
\end{gathered}
$$

Adding this equation and the equation

$$
\begin{aligned}
\left(P^{a}{ }_{j k l}{ }^{\beta} \lambda_{b} p_{\beta}^{b}\right. & \left.-P^{a}{ }_{j l k}^{\beta} \lambda_{b} p_{\beta}^{b}\right)_{; a}=P^{a}{ }_{j k l}{ }^{\beta} ; \lambda_{c} \lambda_{c} p_{\beta}^{c}+P^{a}{ }_{j k l}{ }^{\beta} X S_{b a} p_{\beta}^{b} \\
& -P^{a}{ }_{j k l}^{\beta}{ }^{\beta} I I_{b a}^{0 a} \lambda_{c} p_{a}^{c} p_{\beta}^{b}-(k, l)=-(N-1) P^{0}{ }_{j k l}^{\beta} \lambda_{c} p_{\beta}^{c} \\
& +P^{c}{ }_{j k a}{ }^{a} P^{a}{ }_{b c l}^{\beta} p_{a}^{b} \lambda_{d} p_{\beta}^{d}+P^{a}{ }_{j k l}^{\beta} X S_{b a} p_{\beta}^{b} \\
& +P^{a}{ }_{j k l}{ }^{\beta} I l_{b a}{ }_{b a}^{\alpha} \lambda_{c} p_{a}^{c} p_{\beta}^{b}-(k, l)
\end{aligned}
$$


obtained by the use of (1.19), we get

$$
\begin{aligned}
& \left(X P^{a}{ }_{j k l}+P^{a_{j k l}{ }^{\beta} \lambda_{b}} p_{\beta}^{b}-P^{a}{ }_{j l k}{ }^{\beta} \lambda_{b} p_{\beta}^{b}\right)_{; a} \\
& +(N-2)\left(X P^{0}{ }_{j k l}+\lambda_{a} P^{a}{ }_{j k l}+P^{0}{ }_{j k l}{ }^{\beta} \lambda_{d} p_{\beta}^{d}-P^{0}{ }_{j l k}^{\beta} \lambda_{d} p_{\beta}^{d}\right) \\
& +P^{c}{ }_{j k a}{ }^{\beta}\left(X P^{a}{ }_{b l c}+P^{a}{ }_{b l c}{ }^{\alpha} \lambda_{d} p_{a}^{d}-P^{a}{ }_{b c l}{ }^{\alpha} \lambda_{d} p_{a}^{d}\right) p_{\beta}^{b} \\
& -P^{c}{ }_{j l a}{ }^{\beta}\left(X P^{a}{ }_{b k c}+P^{a}{ }_{b k c}{ }^{\alpha} \lambda_{d} p_{a}^{d}-P^{a}{ }_{b c k}{ }^{\alpha} \lambda_{d} p_{a c}^{d}\right) p_{\beta}^{b} \\
& +X P^{c}{ }_{j k a}{ }^{\beta} P^{a}{ }_{b l c} p_{\beta}^{b}+X P^{c}{ }_{j k l}^{\beta} S_{b c} p_{\beta}^{b}-X P^{c}{ }_{j l a}{ }^{\beta} P^{a}{ }_{b k c} p_{\beta}^{b}-X P^{c}{ }_{j l k}{ }^{\beta} S_{b c} p_{\beta}^{b}=0 .
\end{aligned}
$$

This equation shows that condition (4.8) are obtained from (4.5), (4.6) and covariant derivatives of (4.5),

Thus, as integrability conditions of (4.4), we have only to consider (4.5), (4.6) and their successive derivatives.

We first consider successive derivatives of (4.5), and we shall show that equation

$$
\left.\left(X P^{i}{ }_{j k l}+P^{i}{ }_{j k l}{ }^{\alpha} \lambda_{b} p_{a}^{b}-P^{i}{ }_{j l k}{ }^{\alpha} \lambda_{b} p_{a}^{b}\right)\right|_{n} ^{\beta}=0
$$

obtained form (4.5) by partial differentiation with respect to $p_{\beta}^{n}$ does not give new conditions. Indeed, applying the operator $X$ to both members of (1.20) and using $X \Pi_{b n}^{0 \beta}=\left.\lambda_{b}\right|_{n} ^{\beta}$, we find

$$
\begin{gathered}
X\left(\left.P^{i}{ }_{j k l}\right|_{n} ^{\beta}\right)=X\left(P^{i}{ }_{j k n}{ }^{\beta} ; l\right)+X P^{0}{ }_{j k n}{ }^{\beta} \delta_{l}^{i}-X P^{i}{ }_{j k a}{ }^{\alpha} P^{a}{ }_{b l n}{ }^{\beta} p_{\alpha}^{b}-P^{i}{ }_{j k a}^{\alpha} X P^{a}{ }_{b l n}{ }^{\beta} p_{a}^{b} \\
-X P^{i}{ }_{j k l}{ }^{\alpha} I_{b n}^{\beta}{ }_{b \beta}^{\beta} p_{\beta}^{b}-\left.P^{i}{ }_{j k l}{ }^{\alpha} \lambda_{b}\right|_{n} ^{\beta} p_{\alpha}^{b}-(k, l) .
\end{gathered}
$$

Substituting into this the equation

$$
\begin{aligned}
& X\left(P^{i}{ }_{j k n ; l}^{\beta}\right)- X\left(P^{i}{ }_{j l n}^{\beta} ; k\right)=\left(X P^{i}{ }_{j k n}^{\beta}\right)_{; l}+\lambda_{a} P^{a}{ }_{j k n}{ }^{\beta} \delta_{l}^{i}-P^{i}{ }_{j k l}{ }^{\beta} \lambda_{n} \\
&-\left.P^{i}{ }_{j k n}^{\beta}\right|_{l} ^{\alpha} \lambda_{b} p_{a}^{b}-(k, l) \\
&=\left(X P^{i}{ }_{j k n}{ }^{\beta}\right)_{; l}+\lambda_{a} P^{a}{ }_{j k n}{ }^{\beta} \delta_{l}^{i}-P^{i}{ }_{j k l}{ }^{\beta} \lambda_{n}-P^{i}{ }_{j k l}{ }^{\alpha}{ }_{n}^{\beta} \lambda_{b} p_{a}^{b}-(k, l),
\end{aligned}
$$

we find

$$
\begin{aligned}
& X\left(\left.P^{i}{ }_{j k l}\right|_{n} ^{\beta}\right)+P^{i}{ }_{j k l}^{\alpha}{ }_{n}^{\beta}{ }_{n}^{\beta} \lambda_{b} p_{\alpha}^{b}-P^{i}{ }_{j l k}{ }^{\alpha}{ }_{n}^{\beta} \lambda_{b} p_{\alpha}^{b}+\left.P^{i}{ }_{j k l}^{\alpha}{ }^{\alpha}{ }_{b}\right|_{n} ^{\beta} p_{\alpha}^{b}-\left.P^{i}{ }_{j l k}{ }^{\alpha} \lambda_{b}\right|_{n} ^{\beta} p_{\alpha}^{b} \\
& +P^{i}{ }_{j k l}{ }^{\alpha} \lambda_{b} \delta_{a}^{\beta} \delta_{n}^{b}-P^{i}{ }_{j l k}{ }^{\alpha} \lambda_{b} \delta_{a}^{\beta} \delta_{n}^{b} \\
& =\left(X P^{i}{ }_{j k n}{ }^{\beta}\right)_{; l}+\left(X P^{0}{ }_{j k n}{ }^{\beta}+\lambda_{a} P^{a}{ }_{j k n}{ }^{\beta}\right) \delta_{l}^{i}-X P^{i}{ }_{j k a}{ }^{\alpha} P^{a}{ }_{b l n}{ }^{\beta} p_{\alpha}^{b} \\
& -P^{i}{ }_{j k a}^{\alpha} X P_{b l n}^{a}{ }^{\beta} p_{a}^{b}-X P^{i}{ }_{j k l}{ }^{\alpha} \Pi_{b n}^{0 \beta} p_{a}^{b}-(k, l)
\end{aligned}
$$

or

$$
\begin{aligned}
& \left.\left(X P^{i}{ }_{j k l}+P^{i}{ }_{j k l}{ }^{\alpha} \lambda_{b} p_{\alpha}^{b}-P^{i}{ }_{j l k}^{\alpha}{ }^{\alpha} \lambda_{b} p_{a}^{b}\right)\right|_{n} ^{\beta} \\
& =\left(X P^{i}{ }_{j k n}{ }^{\beta}\right)_{; l}+\left(X P^{0}{ }_{j k n}{ }^{\beta}+\lambda_{a} P^{a}{ }_{j k n}{ }^{\beta}\right) \delta_{l}^{i}-X P^{i}{ }_{j k a}{ }^{\alpha} P^{a}{ }_{b l n}{ }^{\beta} p_{a}^{b} \\
& -P_{j k a}^{i} X P_{b l n}^{a}{ }^{\beta} p_{\alpha}^{b}-X P^{i}{ }_{j k l}{ }^{\alpha} I I_{b n}^{0} p_{a}^{b}-(k, l),
\end{aligned}
$$


which shows that (4.11) is obtained from (4.6), (4.7) and covariant derivatives of (4.6), and consequently that (4.11) is obtained from (4.6) and covariant derivatives of (4.6).

From (4.5) we get

$$
\left(X P^{i}{ }_{j k l}+P^{i}{ }_{j k l}{ }^{\alpha} \lambda_{b} p_{a}^{b}-P^{i}{ }_{j l k}{ }^{\alpha} \lambda_{b} p_{\beta}^{b}\right)_{; m_{1}}=0,
$$

but we can show by a similar method that the partial derivative of this equation with respect to $p_{a}^{i \bar{u}}$ does not give new conditions. From the above equation we obtain

$$
\left(X P^{i}{ }_{j k l}+P^{i}{ }_{j k l}^{\alpha} \lambda_{b} p_{\alpha}^{b}-P^{i}{ }_{j l k}^{\alpha} \lambda_{b} p_{\alpha}^{b}\right)_{; m_{1} ; m_{2}}=0,
$$

but we can show by a similar method that the partial derivative of this equation with respect to $p_{\infty}^{i}$ does not give new conditions.

Repeating this process, we obtain

$$
\left(X P^{i}{ }_{j k l}+P^{i}{ }_{j k l}{ }^{\alpha} \lambda_{b} p_{\alpha}^{b}-P^{i}{ }_{j k l}{ }^{\alpha} \lambda_{b} p_{a}^{b}\right)_{; m_{1} ; m_{2} ; \cdots ; m_{t}}=0, \quad(t=1,2, \cdots) .
$$

We next consider successive derivatives of (4.6). Equation (1.16) shows that, conditions obtained from (4.6) applying first the covariant differentiation and next the partial differentiation and those obtained applying first the partial differentiation and next the covariant differentiation are equivalent.

Thus we shall consider first conditions obtained from (4.6) applying successively only the partial differentiations.

Differentiating partially (4.6) successively, we obtain

$$
\left.\left(X P^{i}{ }_{j k l}^{\alpha}\right)||_{l_{1}}^{\alpha}\right|_{l_{2}} ^{\alpha_{2}}|\cdots|_{l_{s}}^{\alpha}=0, \quad(s=1,2, \cdots) .
$$

But, by virtue of the homogeneity property of $P^{i}{ }_{j k l}{ }^{\alpha}$ with respect to $p_{a}^{i}$, the final equation of (4.14) contains the preceding equations, and consequently equation (4.14) can be written as

$$
\left(X P_{j k l}^{i}{ }_{j k l}^{\alpha}\right)\left|{ }_{l_{1}}^{\alpha}\right| \alpha_{l_{2}}^{\alpha}|\cdots|{ }_{l_{s}^{s}}^{\alpha}=0 \text {. }
$$

Thus the conditions obtained from (4.6) by successive differentiations are

$$
\left[\left.\left(X P^{i}{ }_{j k l}^{\alpha}\right)_{l_{1}}^{\alpha} \alpha_{l_{2}}^{\alpha_{1}}|\cdots|\right|_{l_{s}} ^{\alpha}\right]_{m_{1}}^{\alpha} ; m_{2} ; \cdots ; m_{t}=0 \quad t=0,1,2, \cdots .
$$

Thus we obtain

THEOREM 4.1. In order that (4.1) admit solutions $\xi^{i}(x)$ which are independent of $p_{a}^{i}$ and $\lambda_{j}(x, p)$ which are homogeneous functions of 
degree zero of $p_{\alpha}^{i}$, it is necessary and sufficient that

$$
\left\{\begin{array}{c}
\left(X P^{i}{ }_{j k l}+P^{i}{ }_{j k l}^{\alpha} \lambda_{b} p_{a}^{b}-P^{i}{ }_{j l k}^{\alpha} \lambda_{b} p_{a}^{b}\right)_{; m_{1} ; m_{2} ; \cdots ; m_{p}}=0, \\
\left.\left(\left.\left.X P^{i}{ }_{j k l}^{\alpha}\right|_{l_{1}} ^{\alpha}\right|_{l_{2}} ^{\alpha_{2}}|\cdots|{ }_{l_{s}}^{\alpha}\right)_{j}\right) ; m_{1} ; m_{2} ; \cdots ; m_{t}=0, \\
(p, t=0,1,2, \cdots)
\end{array}\right.
$$

are algebraically consistent in $\xi^{i}, \xi^{i} ;$ and $\lambda_{j}$.

Since these equations are linear homogeneous in $\xi^{i}, \xi^{i} ; j$ and $\lambda_{j}$, we get from this,

THEOREM 4.2. In order that the space of $K$-spreads admit $r$ linearly independent infinitesimal projective collineations, it is necessary and sufficient that (4.17) contain $N(N+2)-r$ linearly independent equations and others be consequences of others.

If we denote such $r$ linearly independent infinitesimal projective collineations by

$$
\bar{x}^{i}=x^{i}+\xi_{\mathrm{A}}^{i}(x) d t, \quad(A=1,2, \cdots r),
$$

then, any $\xi^{i}$ satisfying (4.1) is a linear combination of $\xi_{A}^{i}$ with constants coefficients. But, from Theorem 2.1 and 3.1, we know that $X_{B} \xi_{C}^{i}$ also satisfy (4.1) and consequently, $X_{B} \xi_{C}^{i}$ should be linear combinations of $\xi_{A}^{i}$ with constant coefficients. Thus, from Theorem 3.7, we have

THEOREM 4.3. In order that the space of $K$-spreads admit an $r$ parameter group of projective collineations, it is necessary and sufficient that (4.17) contain $N(N+2)-r$ linearly indepent equations and the others be consquences of these.

If (4.1) are completely integrable, then (4.5) and (4.6) should be identities in $\xi^{i}, \xi_{;}^{i} ;$ and $\lambda_{j}$, and consequently we must have $P^{i}{ }_{j k l}=0$ and $P^{i}{ }_{j k l}{ }^{\alpha}=0$. Thus we have

THEOREM 4.4. In order that an $N$-dimensional space of $K$-spreads admit a group of projective collineations of the maximum order $N(N+2)$, it is necessary and sufficient that the space be projectively flat.

University of Tokyo and University of Kumamoto. 


\section{Bibliography}

1. R.S. Clark: Projective collineations in a space of $K$-speads. Proc. Cambridge Phil. Soc., 41 (1945), 210-223.

2. E.T. Davies: On the isomorphic transformations in a space of $K$-speads. The Journal of the London Math. Soc., 18 (1943), 100-107.

3. J. Douglas: The general geometry of paths. Ann. of Math., 29 (1928), 143-168.

4. $\quad$ Systems of $K$-dimensional manifolds in an $N$-dimensional space. Math. Ann., 105 (1931), 707-733.

5. L. P. Eisenhart: Continuous groups of transformations. (1933), Princeton University Press.

6. L.P. Eisenhart and M.S. Knebelman: Displacements in a geometry of paths which carries paths into paths. Proc. Nat. Acad. Sci. U.S. A. 13 (1927), 38-42.

7. H. Hiramatu: On projective collineations in a space of hypersurfaces. Tensor, 2 (1952), 1-14.

8. M. S. Knebelman : Collineations and motions in generalized spaces. Amer. Journal of Math., 51 (1929), 527-564.

9. J. A. Schouten and E. R. van Kampen: Beiträge zur Theorie der Deformation. Prace Mat. Fiz., 41 (1931), 1-19.

10. W. Slebodzinski: Sur les transformations isomorphiques d'une variété à connexion affine. Ibidem, 39 (1932), 55-62.

11. Buchin Su: Descriptive collineations in spaces of $K$-spreads. Trans. Amer. Math. Soc., 61 (1947), 495-507.

12. : On isomorphic transformations of $K$-spreads in a Douglas space. Science Record, Academia Sinica, 2 (1947), 15-19; II 2 (1948), 139-146.

13. - A characterization property of affine collineations in a space of $K$-spreads. Bull. Amer. Math. Soc., 54 (1948), 136-138.

14. - A generalization of descriptive collineations in a space of $K$-spreads. The Journal of the London Math. Soc., 25 (1950), 236-238.

15. K. Yano: Groups of transformations in generalized spaces, (1949), Tokyo.

16. K. Yano and H. Hiramatu: On projective geometry of $K$-spreads. Compositio Mathematica, 10 (1952), 286-296. 